\title{
Stability and Scalability of Homogeneous Vehicular Platoon: Study on the Influence of Information Flow Topologies
}

\author{
Yang Zheng, Shengbo Eben Li, Jianqiang Wang, Dongpu Cao, and Keqiang Li
}

\begin{abstract}
In addition to decentralized controllers, the information flow among vehicles can significantly affect the dynamics of a platoon. This paper studies the influence of information flow topology on the internal stability and scalability of homogeneous vehicular platoons moving in a rigid formation. A linearized vehicle longitudinal dynamic model is derived using the exact feedback linearization technique, which accommodates the inertial delay of powertrain dynamics. Directed graphs are adopted to describe different types of allowable information flow interconnecting vehicles, including both radar-based sensors and vehicle-to-vehicle (V2V) communications. Under linear feedback controllers, a unified internal stability theorem is proved by using the algebraic graph theory and Routh-Hurwitz stability criterion. The theorem explicitly establishes the stabilizing thresholds of linear controller gains for platoons, under a large class of different information flow topologies. Using matrix eigenvalue analysis, the scalability is investigated for platoons under two typical information flow topologies, i.e., 1) the stability margin of platoon decays to zero as $0\left(1 / N^{2}\right)$ for bidirectional topology; and 2$)$ the stability margin is always bounded and independent of the platoon size for bidirectional-leader topology. Numerical simulations are used to illustrate the results.
\end{abstract}

Index Terms-Autonomous vehicles, information flow topology, platoon, scalability, stability.

\section{INTRODUCTION}

$\mathbf{P}$ LATOONING of road vehicles provides a promising solution to several critical issues of today's road transportation due to its potential to significantly increase highway capacity, enhance safety, and reduce fuel consumption, as well as $\mathrm{CO} 2$ emission [1]-[3]. The objective of platoon control is to ensure that all vehicles in a platoon move at the same speed while maintaining a desired formation geometry, which is specified by a desired inter-vehicle spacing policy. Control design of a

Manuscript received July 17, 2014; revised November 2, 2014 and January 7 , 2015; accepted February 1, 2015. This work was supported by the NSF of China under Grant 51205228 , by the Tsinghua University Initiative Scientific Research Program under Grant 2012THZ0, and by the Chinese National Programs for High Technology Research and Development under Grant 2012AA111901. The Associate Editor for this paper was J. Ploeg. (Yang Zheng and Shengbo Eben Li contributed equally to this work.)

Y. Zheng, S. E. Li, J. Wang, and K. Li are with the State Key Laboratory of Automotive Safety and Energy, Department of Automotive Engineering, Tsinghua University, Beijing 100084, China (e-mail: zhengy13@mails.tsinghua. edu.cn; lisb04@gmail.com; wjqlws@ @tsinghua.edu.cn; likq@tsinghua.edu.cn).

D. Cao is with the Department of Automotive Engineering, Cranfield University, Bedford MK43 0AL, U.K. (e-mail: d.cao@cranfield.ac.uk).

Color versions of one or more of the figures in this paper are available online at http://ieeexplore.ieee.org.

Digital Object Identifier 10.1109/TITS.2015.2402153 platoon has a long history that dates back to the mid-sixties of the last century [4]. It has recently attracted extensive research interests, see [5]-[10] and the references therein.

From the viewpoint of control, a platoon system can be considered as a combination of vehicle longitudinal dynamics, information exchange flow, decentralized controllers and intervehicle spacing policies [6], [7], [9]. The vehicle longitudinal dynamics depict the behavior of each vehicle in longitudinal direction. The platoon is said to be "homogeneous" if all vehicles have identical dynamics; otherwise it is called "heterogeneous" [10]. The information exchange flow defines how the vehicles in a platoon exchange information with each other, including the exchanged information and information flow topologies among vehicles. Decentralized controllers implement specific feedback control laws for each vehicle. Most common control laws are linear, for comprehensive results on theoretical analysis and design methods, and convenience in hardware implementations [7], [10]. The available information to each controller is often limited to a neighboring region because of the range limitation of sensing and communication systems. As a result, controllers use only local information to achieve a global performance for the platoon. The spacing policy sets rules of the desired distance between two adjacent vehicles, and further dictates the desired formation geometry for the platoon. Here, we focus on a homogenous vehicular platoon with rigid formation and linear feedback controllers, as used by Seiler [9], Barooah [11], and Darbha [12].

The information flow topology applied in a platoon is closely related to the way a vehicle acquires the information of its surrounding vehicles. Early-stage platoons are mainly radarbased without widely using the inter-vehicle communications. This means that a vehicle can only obtain the information of its nearest neighbors, i.e., front and back vehicles [7], [13], [14]. Under the radar-based sensing framework, the commonly used information flow topologies include the predecessor following type, the predecessor-leader following type, and the bidirectional type [5], [9], [13]-[15]. Note that the predecessor-leader following type needs a leader with information broadcasting functions. Their relationship with string stability was studied by Darbha and Hedrick [6], [19], Seiler [9], and Ploeg [10], [17], [18] etc. Darbha et al. pointed out that under the constantdistance policy, a predecessor following-type platoon using identical linear controllers cannot guarantee string stability because its associated denominator polynomial has at least an instability root [6]. Seiler et al. showed that there was an 
essential limitation with localized linear controllers using the constant distance policy and predecessor following type, since small spacing errors acting on one vehicle can be amplified along the vehicle string due to a complementary sensitivity integral constraint [9]. Four major approaches have been proposed to improve the string stability. One approach is to use non-identical controllers to achieve bounded stability, but at the expense that the controller gains must increase linearly with respect to the platoon scale [16]. The second approach is to broadcast the leader information to every following vehicle, resulting in the aforementioned predecessor-leader following topology [6], [9]. This topology inevitably introduces certain time delays because it needs to transmit information from the leader to all the following vehicles. The third approach is to relax the formation rigidity by using the constant time headway policy instead of the constant distance policy [19], [20]. The last approach is to extend the information flow topology to the bidirectional type, e.g., [7], [9], [14], [21], [22], [25] or to multiple-vehicle look-ahead type, e.g., [18]. For the bidirectional type, two radars are installed on each vehicle, front and back, to detect its adjacent two vehicles. The controller then can use the information of both its preceding vehicle and following vehicle for control. Recently, several rigorous analyses for string stability are provided. For example, Naus et al. derived a necessary and sufficient frequency domain condition for platoon string stability, and experimented with two vehicles equipped with cooperative adaptive cruise control [10]. Ploeg et al. proposed a novel definition for string stability on the basis of the notion of $L_{p}$ stability [17], which could apply to both linear and nonlinear platoon system. Several other recent research on string stability can be found in [23] and [24].

Although extensive research has been conducted on radarbased topologies, more information flow topologies have emerged with the rapid deployment of vehicle-to-vehicle (V2V) communications such as DSRC, VANET, and MANET [26], [27]. The V2V communication generates various information flow topologies, including the two-predecessors following type, two-predecessor-leader following type and $h$-predecessors following type, etc. [28]. A few studies have been conducted to examine their influence on platoon performance, including stability and scalability. For example, Darbha et al. pointed out that at least one vehicle should communicate to a large number of other vehicles if the spacing errors in the platoon need to be guaranteed insensitive to the platoon size [12]. Darbha and Pagilla investigated the limitations of employing undirected information flow to maintain a rigid formation, and indicated that there was a critical value of platoon scale beyond which the motion would lose stability [29]. Fax et al. used the eigenvalues of the Laplacian matrix to determine the formation stability, and proved that formation stability could be decomposed into two components: i.e., stability of information flow for the given graph and stability of individual vehicles for the given controller [30]. Ploeg et al. developed a $\mathcal{H}_{\infty}$ controller synthesis approach to guarantee string stability, and designed controllers for platoons under one- and two- vehicle look-ahead information flow topologies [18].

This paper further studies the influence of different information flow topologies on the internal stability and scalability of a platoon of homogenous vehicles moving in a rigid formation. The vehicle dynamics are linearized using exact feedback linearization technique, and the linear feedback law is limited to proportional type, which were used in many previous studies, e.g., [20]-[22], [25], [32]. The constant distance policy is employed for the high achievable traffic flow. The main contributions of this paper are:

1) A unified internal stability theorem is derived by using the algebraic graph theory and Routh-Hurwitz stability criterion, which explicitly establishes the stabilizing thresholds of the linear controller gains. The internal stability theorem is suitable for a large class of information flow topologies, either radar-based or communication-based. This theorem is actually an extension of the main result in Ghasemi et al. [21], [22]. The main result in [21] and [22] was derived from another approach, called partial differential equation approximation, but its application was limited to bidirectional topology and bidirectionalleader topology.

2) The scalability of platoons under two typical information flow topologies is investigated by using matrix eigenvalue analysis, and it is proved that: 1) the real part of least stable closed-loop eigenvalue decays to zero as $0\left(1 / N^{2}\right)$ ( $N$ denotes the number of following vehicles) for bidirectional topology; 2) the stability margin is always bounded away from zero and independent of the platoon size $N$ for bidirectional-leader topology. This finding is actually an extension of [25], [31] and [32], in which the inertial delay of vehicle powertrain had not been considered, and the dynamics of each vehicle were simplified to be ideal double integrators.

The remainder of this paper is organized as follows: Section II introduces the problem of platoon control, including graph-based modeling of different types of information flow topologies. Section III presents two theorems for homogeneous platoon, i.e., 1) the internal stability theorem with different information flow topologies; 2) the scalability theorem with two typical information flow topologies. Numerical simulations are shown in Section IV. Section V is for concluding remarks.

\section{Problem Statement}

The platoon has $N+1$ vehicles, shown in Fig. 1, including a leading vehicle (noted as the leader) and $N$ following vehicles (noted as the followers). The platoon runs on a flat road, and can have different information flow topologies, either radar-based or communication-based. Fig. 1 shows six kinds of commonly used topologies, including:

1) Predecessor following (PF) topology;

2) Predecessor-leader following (PLF) topology;

3) Bidirectional (BD) topology;

4) Bidirectional-leader (BDL) topology;

5) Two-predecessors following (TPF) topology;

6) Two-predecessor-leader following (TPLF) topology.

For conciseness, many other topologies are not exhibited here, but they all can be analyzed using similar approaches. Note 


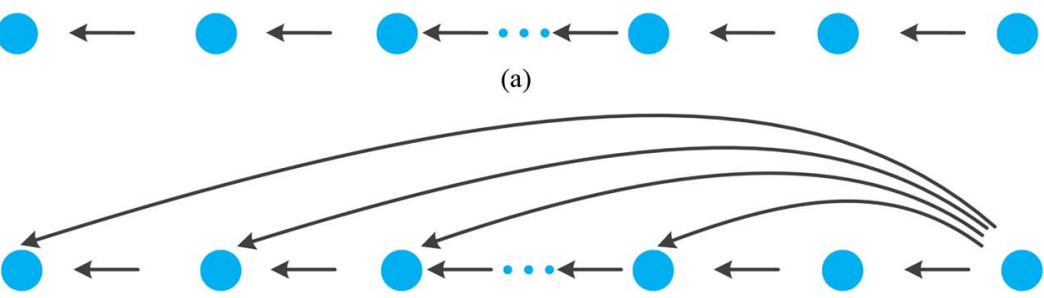

(b)

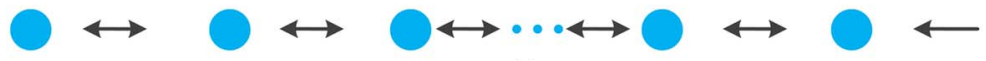

(c)

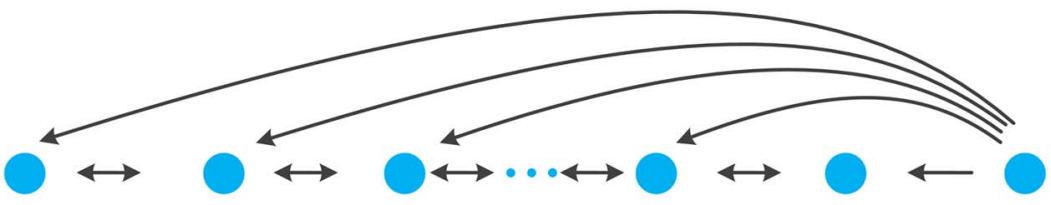

(d)

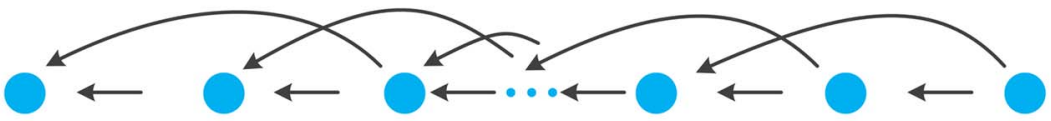

(e)

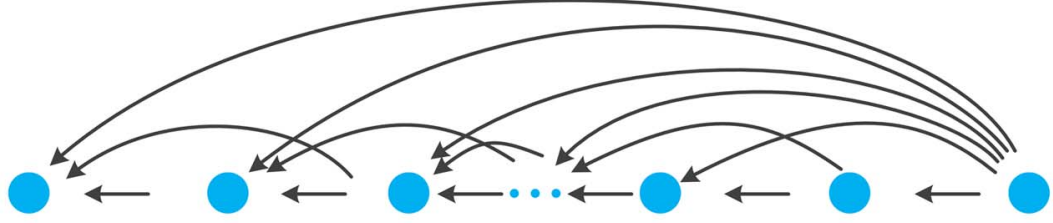

(f)
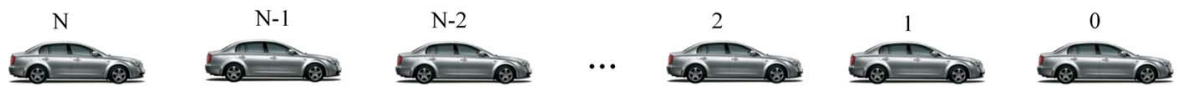

Fig. 1. Typical information flow topologies for platoons. (a) PF. (b) PLF. (c) BD. (d) BDL. (e) TPF. (f) TPLF.

that the exchanged information can contain all the subjected vehicle's position, velocity, and acceleration, or some of them.

Notations: Most of the notations in this paper are fairly standard. For convenience, some notations are explained here. The real and complex domains are denoted by $\mathbb{R}$ and $\mathbb{C}$, respectively. The real part of a complex number $s \in \mathbb{C}$ is denoted by $\operatorname{Re}(s)$, and the imaginary part by $\operatorname{Im}(s)$. The set of $m \times n$ real matrices is denoted by $\mathbb{R}^{m \times n}$. The transpose of a vector or a matrix $A$ is noted by $A^{T}$. We define $\mathbb{C}_{-}=\{s \in \mathbb{C} \mid \operatorname{Re}(\mathrm{s}) \leq 0\}$ and $\mathbb{C}_{-}=$ $\{s \in \mathbb{C} \mid \operatorname{Re}(s)<0\}$. We use $I_{n}$ as the identity matrix of dimension $n$. Let $\sigma_{i}(A)$ denote the $i$-th eigenvalue of matrix $A \in$ $\mathbb{R}^{n \times n}, i=1,2, \cdots, n$, and all its eigenvalues are represented in an increasing order of their real parts, i.e., $\operatorname{Re}\left(\sigma_{1}(A)\right) \leq$ $\operatorname{Re}\left(\sigma_{2}\right) \leq \cdots \leq \operatorname{Re}\left(\sigma_{n-1}\right) \leq \operatorname{Re}\left(\sigma_{n}(A)\right)$. The spectrum of $A$ is denoted by $S(A)=\left\{\sigma_{1}(A), \cdots, \sigma_{n}(A)\right\}$. A matrix $A \in$ $\mathbb{R}^{n \times n}$ is called Hurwitz (or stable) if and only if all its eigenvalues have negative real part, i.e., $\sigma_{1}(A) \in \mathbb{C}_{-}, i=1,2, \cdots, N$. $\operatorname{diag}\left\{a_{1}, a_{2}, \cdots, a_{n}\right\}$ denotes a diagonal matrix whose diagonal entries starting at the upper left corner are $a_{1}, a_{2}, \cdots, a_{n}$. Let $A \in \mathbb{R}^{m \times n}, B \in \mathbb{R}^{p \times q}$, then $A \otimes B$ is the Kronecker product of $A$ and $B$

$$
A \otimes B=\left[\begin{array}{ccc}
a_{11} B & \cdots & a_{m 1} B \\
\vdots & \ddots & \vdots \\
a_{1 n} B & \cdots & a_{m n} B
\end{array}\right] \in \mathbb{R}^{m p \times n q} .
$$

A real scalar-valued function of time, $x: \mathbb{R} \rightarrow \mathbb{R}$, is denoted by $x(t) \in \mathbb{R}$ and sometimes simply denoted as $x$. Notation $O(\epsilon)$ is used to denote the infinitesimals of the same order as $\epsilon$.

\section{A. Model for Vehicle Longitudinal Dynamics}

A platoon can be viewed as a collection of nodes, i.e., vehicles. For each vehicle, its longitudinal dynamics include the engine, drive line, brake system, aerodynamics drag, tire friction, rolling resistance, and gravitational force, etc. Some reasonable assumptions should be used to obtain a concise model for control [19], [20], [33], [34]:

1) The tire longitudinal slip is negligible, and the powertrain dynamics are lumped into a first-order inertial transfer function;

2) The vehicle body is considered to be rigid and symmetric;

3) The influence of pitch and yaw motions is neglected;

4) The driving and braking torques are controllable inputs.

The vehicle longitudinal dynamics are simplified, but still nonlinear, as follows:

$$
\left\{\begin{array}{l}
\dot{s}_{i}(t)=v_{i}(t) \\
\dot{v}_{i}(t)=\frac{1}{m_{i, v e h}}\left(\eta_{T, i} \frac{T_{i}(t)}{R_{i}}-C_{A, i} v_{i}^{2}-m_{i, v e h} g f\right), \\
\tau_{i} \dot{T}_{i}(t)+\left(T_{i}(t)=T_{i, \text { des }}(t)\right. \\
\quad \quad i=1,2 \cdots, N
\end{array}\right.
$$


where $s_{i}(t), v_{i}(t)$ denote the position and velocity of vehicle $i$, $m_{i, v e h}$ is the vehicle mass, $C_{A, i}$ is the lumped aerodynamic drag coefficient, $g$ is the acceleration due to gravity, $f$ is the coefficient of rolling resistance, $T_{i}(t)$ denotes the actual driving/braking torque, $T_{i, \text { des }}(t)$ is the desired driving/braking torque, $\tau_{i}$ is the inertial delay of vehicle longitudinal dynamics, $R_{i}$ denotes the tire radius, and $\eta_{T, i}$ is the mechanical efficiency of driveline. The position and velocity of the leading vehicle are denoted by $s_{0}(t)$ and $v_{0}(t)$, respectively.

The exact feedback linearization technique is used to convert the nonlinear model into a linear one for controller design. The same technique has been widely used before, e.g., [7], [14], [20] and [21]. The output of position with relative degree three is used to construct the feedback linearization law, as shown in (3)

$$
T_{i, \text { des }}(t)=\frac{1}{\eta_{T, i}}\left(C_{A, i} v_{i}\left(2 \tau_{i} \dot{v}_{i}+v_{i}\right)+m_{i, v e h} g f+m_{i, v e h} u_{i}\right) R_{i},
$$

where $u_{i}$ is the new input signal after linearization. Then, we obtain a linear model for vehicle longitudinal dynamics

$$
\tau_{i} \dot{a}_{i}(t)+a_{i}(t)=u_{i}(t),
$$

where $a_{i}(t)=\dot{v}_{i}(t)$ denotes the acceleration of vehicle $i$. For platoon control, a 3rd-order state space model is derived for each vehicle:

$$
\dot{x}_{i}(t)=A_{i} x_{i}(t)+B_{i} u_{i}(t),
$$

where

$$
x_{i}(t)=\left[\begin{array}{c}
s_{i} \\
v_{i} \\
a_{i}
\end{array}\right], A_{i}=\left[\begin{array}{ccc}
0 & 1 & 0 \\
0 & 0 & 1 \\
0 & 0 & -\frac{1}{\tau_{i}}
\end{array}\right], B_{i}=\left[\begin{array}{c}
0 \\
0 \\
\frac{1}{\tau_{i}}
\end{array}\right] .
$$

\section{B. Model for Information Flow}

The information flow topology describes the information used by each local controller, and has significant influence on the collective behavior of the platoon. Moreover, some properties (e.g., stability and scalability) are not only related to decentralized controllers, but also depend on the information flow topology [9], [12], [29], [30], [35]. Here, directed graphs are adopted to develop a unified model for allowable information flow that interconnect vehicles in a platoon, including all aforementioned topologies.

The platoon includes $N$ followers and one leader. The information flow among followers is modeled by a directed graph topology $G=\{V, E\}$ with $N$ nodes $V=\left\{\alpha_{1}, \alpha_{2}, \cdots, \alpha_{N}\right\}$, and a set of edges $E=V \times V$. The node $\alpha_{i}$ represents the $i$-th following vehicle in a platoon whose dynamics are described by (5), and each edge represents a directional information exchange between two vehicles. To model the information flow from the leader to followers, we define an augmented graph as $\tilde{G}=\{\tilde{V}, \tilde{E}\}$, where $\tilde{V}=\left\{\alpha_{0}, \alpha_{1}, \ldots, \alpha_{N}\right\}$ is the node set including both the leader and the followers and $\tilde{E}=\tilde{V} \times \tilde{V}$ is the set of edges including the information flow both among followers and from the leader to followers. The properties of information flow modeled by the directed graphs $G$ and $\tilde{G}$ can be represented by three matrices:

1) Adjacent matrix $M$;

2) Laplacian matrix $L$;

3) Pinning matrix $P$.

The method that uses matrices to study graphs is known as algebraic graph theory [30], [36]. The adjacent matrix associated with graph $G$ is defined as $M=\left[m_{i j}\right] \in \mathbb{R}^{N \times N}$ with each entry defined as

$$
\left\{\begin{array}{l}
m_{i j}=1, \quad \text { if }\left\{\alpha_{j}, \alpha_{i}\right\} \in E \\
m_{i j}=0, \quad \text { if }\left\{\alpha_{j}, \alpha_{i} \notin E\right.
\end{array},\right.
$$

where $\left\{\alpha_{j}, \alpha_{i}\right\} \in E$ means there is a directional edge from vehicle $j$ to vehicle $i$, i.e., vehicle $i$ can obtain the information of vehicle $j$. It is assumed that there is no self-loop, i.e., $m_{i i}=0$. The Laplacian matrix $L=\left[l_{i j}\right] \in \mathbb{R}^{N \times N}$ associated with graph $G$ is defined as

$$
l_{i j}=\left\{\begin{array}{ll}
-m_{i j}, & i \neq j \\
\sum_{k=1}^{N} m_{i k}, & i=j
\end{array} .\right.
$$

The pinning matrix $P$ associated with the augmented graph $\tilde{G}$ represents the information flow from the leader to followers, defined as:

$$
P=\operatorname{diag}\left\{p_{1}, p_{2}, \ldots, p_{N}\right\},
$$

where $p_{i}=1$ if edge $\left\{\alpha_{0}, \alpha_{i}\right\} \in \tilde{E} ; p_{i}=0$ otherwise. The expression $\left\{\alpha_{0}, \alpha_{i}\right\} \in \tilde{E}$ means that vehicle $i$ can receive information from the leader. The weight $p_{i}$ has been called pinning gains in the field of complex networks [37]. If $p_{i}=1$, vehicle $i$ is said to be pinned to the leading vehicle. We define leader accessible set of node $i$ as

$$
\mathbb{P}_{i}=\left\{\begin{array}{ll}
\{0\}, & \text { if } p_{i}=1 \\
\emptyset & \text { if } p_{i}=0
\end{array} .\right.
$$

Several definitions associated with graph topology $\tilde{G}$ should be stated for completeness [36]:

1) Directed path. A directed path of length $\zeta+1$ from node $\alpha_{i}$ to node $\alpha_{j}$ is an ordered set of distinct nodes $\left\{\alpha_{i}, \alpha_{i_{1}}\right.$, $\left.\ldots, \alpha_{i_{\zeta}}, \alpha_{j}\right\}$ such that $\left\{\alpha_{i}, \alpha_{i_{1}}\right\} \in \tilde{E},\left\{\alpha_{i_{\zeta}}, \alpha_{j}\right\} \in \tilde{E}$ and $\left\{\alpha_{i_{k}}, \alpha_{i_{k+1}}\right\} \in \tilde{E}$ for all $k \in\{1,2, \ldots, \zeta-1\}$ and $\zeta<N$.

2) Spanning tree. A spanning tree is a tree formed by some or all the edges of graph that connect all the nodes of the graph. The graph $\tilde{G}$ is said to have a spanning tree if a subset of the edges forms a spanning tree.

3) Neighbor set. Vehicle $j$ is said to be a neighbor of vehicle $i$ if $m_{i j}=1$, which means vehicle $i$ can obtain information from vehicle $j$ by $\mathrm{V} 2 \mathrm{~V}$ communication or by radar-based detection. The neighbor set of vehicle $i$ is denoted by $\mathbb{N}_{i}=$ $\left\{j \mid m_{i j}=1\right\}$.

Here, it is assumed that the augmented graph $\tilde{G}$ contains at least one spanning tree rooting from the leader [38]. In other words, there exists a directed path (not necessarily unique) from the leader to every following vehicle, which implies that every 
follower can obtain the leader information directly or indirectly. It is obvious that all the information flow topologies shown in Fig. 1 satisfy the assumption of containing a spanning tree.

\section{Formulation of Closed-Loop Platoon Dynamics}

In engineering practice, both vehicle dynamics and platoon controllers can be different from each other, which imply that the platoon is heterogeneous. However, a platoon is often formed by the vehicles of the same-type, e.g., either by only trucks or by only passenger cars. In such cases, vehicle dynamics are close to each other. In this paper, it is assumed that the platoon is homogeneous, i.e., $A_{i}=A, B_{i}=B(i=$ $1,2, \cdots, N)$, and their controllers are designed to be identical, as studied in [9], [11], and [12]. The leading vehicle is considered to be of constant-velocity type, i.e., $s_{0}=v_{0} t$. The objective of platoon control is to track the speed of the leading vehicle while maintaining a rigid formation governed by the constant distance policy between any two consecutive vehicles, i.e.,

$$
\left\{\begin{array}{l}
v_{i}(t)=v_{0}(t) \\
s_{i-1}(t)-s_{i}(t)=d_{i-1, i}
\end{array} \quad, i=1,2, \cdots N,\right.
$$

where $d_{i-1, i}$ is the desired constant spacing between vehicle $i-1$ and vehicle $i$. There are two major spacing policies for vehicular platoons: the constant distance (CD) policy and constant time headway $(\mathrm{CTH})$ policy [5], [19]. In the CD policy, the desired distance between two consecutive vehicles is independent of vehicle velocity, which can lead to a very high traffic capacity. For the CTH policy, the desired intervehicle range varies with vehicle velocity, which accords with driver behaviors to some extent but limits the achievable traffic capacity. Here, we only consider the CD policy, which means that the vehicles are controlled to move in a rigid formation while following a leading vehicle. Note that $d_{i-1, i}$ contains the length of the vehicle body.

The controllers are distributed in each vehicle, and each controller can only use its neighborhood information specified by $\mathbb{I}_{i}=\mathbb{N}_{i} \cup \mathbb{P}_{i}$. The linear control law of each vehicle is

$$
u_{i}(t)=-\sum_{j \in \mathbb{I}_{i}}\left[k_{1}\left(s_{i}-s_{j}-d_{i, j}\right)+k_{2}\left(v_{i}-v_{j}\right)+k_{3}\left(a_{i}-a_{j}\right)\right] \text {, }
$$

where $k_{\#}(\#=1,2,3)$ is the control gain of the linear controller. The augmented graph $\tilde{G}$ specifies the information flow topology. A control law satisfying (10) is said to have structure $\tilde{G}$, whereas an unstructured control law is one that has structure corresponding to the complete graph which requires communication between any pair of vehicles. Many existing literature on platoon analysis belongs to the study of structured control law in an explicit or implicit way, see [6], [17], [18], and [20] for examples. It should be noted that we are only interested in static and linear control laws using the graph $\tilde{G}$. Thus, a communication link, if it exists, is assumed to be perfect in the sense that we ignore the effects such as quantization issues, data dropouts and time delays.

The desired trajectory of the $i$-th vehicle is

$$
s_{i}^{*}=s_{0}-d_{0, i}=s_{0}-\sum_{j=0}^{i-1} d_{j, j+1} .
$$

To rewrite (10) into collective form, we define three new tracking errors $\tilde{s}_{i}, \tilde{v}_{i}$ and $\tilde{a}_{i}$

$$
\left\{\begin{array}{l}
\tilde{s}_{i}=s_{i}-s_{i}^{*} \\
\tilde{v}_{i}=v_{i}-\dot{s}_{i}^{*}=v_{i}-v_{0} \\
\tilde{a}_{i}=a_{i}-\ddot{s}_{i}^{*}=a_{i}
\end{array} .\right.
$$

For each vehicle, we can lump its tracking error with neighborhood vehicles specified by $\mathbb{I}_{i}$. The lumped tracking error is

$$
\varepsilon_{i}=\sum_{j \in \mathbb{I}_{i}}\left(\tilde{x}_{i}-\tilde{x}_{j}\right),
$$

where $\tilde{x}_{i}=\left[\tilde{s}_{i}, \tilde{v}_{i}, \tilde{a}_{i}\right]$. Substituting (13) into (10), the control law is rewritten into a compact form:

$$
u_{i}(t)=-k^{T} \varepsilon_{i}(t),
$$

where $k=\left[k_{1}, k_{2}, k_{3}\right]^{T}$. Then, the closed-loop dynamics of vehicle $i$ becomes

$$
\begin{aligned}
\dot{\tilde{x}}_{i} & =A \tilde{x}_{i}-B k^{T} \varepsilon_{i}(t) \\
& =A \tilde{x}_{i}-B k^{T}\left[\sum_{j=1}^{N} m_{i j}\left(\tilde{x}-\tilde{x}_{j}\right)+p_{i}\left(\tilde{x}_{i}-\tilde{x}_{0}\right)\right] .
\end{aligned}
$$

For the closed-loop dynamics of the homogeneous platoon, we define the collective states of all vehicles as

$$
X=\left[\tilde{x}_{1}, \tilde{x}_{2}, \cdots, \tilde{x}_{N}\right]^{T} .
$$

Hence, the unified overall closed-loop dynamics of the platoon interconnected by a given information exchange topology are written in the following compact form

$$
\dot{X}=\left\{I_{N} \otimes A-(L+P) \otimes B k^{T}\right\} X .
$$

The overall closed-loop system matrix is

$$
A_{c}=I_{N} \otimes A-(L+P) \otimes B k^{T} .
$$

From (17), it is clear that the platoon dynamics are a function of vehicle longitudinal dynamics (denoted by $A, B$ ), the information flow topologies (denoted by matrix $L+P$ ) and decentralized feedback control law (denoted by controller gain $k^{T}$ ). The overall closed-loop system matrix $A_{c}$, shown in (18), reflects the local vehicle closed-loop matrix $A-B k^{T}$ as modified on the information flow topology $L+P$. Therefore, the stability of a platoon depends not only on its decentralized controllers but also on the information flow topologies. Moreover, the information flow can cast fundamental limitations for certain platoon properties, i.e., stability and scalability. This paper focuses on the influence of different information flow topologies on the internal stability of platoon, and the topology in each case is fixed. For switching topology case, the matrix $L+P$ is time-varying rather than fixed. In Section III, the internal stability and scalability under different information flow topologies will be analyzed based on (17) through the algebraic graph theory, Routh-Hurwitz stability criterion and matrix eigenvalue analysis. 


\section{Closed Loop Stability With Different INFORMATION FLOW TOPOLOGIES}

This section focuses on the stability analysis of homogeneous platoons in a rigid formation. It should be noted that there are two kinds of stability for platoons, i.e.,

1) Internal stability. A platoon with linear time-invariant dynamics is said to be internal stable if and only if all real parts of the eigenvalues of the closed-loop system are negative [21], [25].

2) String stability. A platoon is said to be string stable if and only if the disturbances are not amplified when propagating along the vehicle string [6], [9], [17].

This paper only considers the internal stability and leaves the string stability for future discussion. In this section, two theorems will be proved for homogeneous platoons under different information flow topologies. One is about the unified internal stability of platoons under a large class of information flow topologies. The other one is about the scalability of platoons under two typical topologies.

\section{A. Internal Stability Analysis for Different Information Flow Topologies}

Before presenting the internal stability theorem, we need the following Lemmas.

Lemma 1. [39]: Let a matrix $Q=\left[q_{i j}\right] \in \mathbb{R}^{n \times n}$. Then all the eigenvalues of $Q$ are located in the union of the n disks

$$
\bigcup_{i=1}^{n}\left\{\lambda \in \mathbb{C}|| \lambda-q_{i i}\left|\leq \sum_{j=1, j \neq i}^{n}\right| q_{i j} \mid\right\} \text {. }
$$

Lemma 2. [40]: Let a matrix $Q=\left[q_{i j}\right] \in \mathbb{R}^{n \times n}$ and

$$
J=\left\{i \in\{1,2, \cdots, n\}|| q_{i i}\left|>\sum_{j=1, j \neq i}\right| q_{i j} \mid\right\} \neq \emptyset .
$$

If for each $i \in J$, there is a sequence of nonzero elements of $\mathrm{Q}$ of the form $\left\{q_{i i_{1}}, q_{i_{1} i_{2}}, \cdots, q_{i_{r} j}\right\}$ with $j \in J$, then $\mathrm{Q}$ is nonsingular.

Lemma 3. [30]: Let $\lambda_{i}, i=1,2, \ldots, N$ be the eigenvalues of $L+P$, which may or may not be distinct. The platoon dynamics (17) are asymptotically stable if and only if all the matrices

$$
A-\lambda_{i} B k^{T}, \quad i=1,2, \cdots, N
$$

are Hurwitz. Moreover, we have

$$
S\left(A_{c}\right)=\bigcup_{i=1}^{n} S\left(A-\lambda_{i} B k^{T}\right) .
$$

Lemma 1 is the well-known Geršgorin Disk Criterion. The first result of this paper is stated as follows.

Theorem 1: Consider the homogeneous platoon using linear controllers with the closed-loop dynamics as in (17). Let $\lambda_{i}, i=1,2, \ldots, N$ be the eigenvalues of $L+P$. The following statements hold:

1.1) All the eigenvalues of $L+P$ are located in the open right-half plane, i.e., $-\lambda_{i} \in \mathbb{C}, i=1,2, \ldots, N$, when graph $\tilde{G}$ contains a spanning tree.

1.2) All the eigenvalues of $L+P$ are real numbers, i.e., $\lambda_{i} \in \mathbb{R}, i=1,2, \ldots, N$, if graph $G$ satisfies one of the following conditions, no matter how many followers are pinned to the leader. (Note that the parameter $h$ in the following conditions a) and b) denotes the number of nodes which the $i$-th node can reach.)

a) Followers in a platoon are of "look-ahead" type, i.e., $\mathbb{N}_{i}=\{i-h, \cdots, i-1,\} \cap\{1, \cdots, N\}$.

b) Followers in a platoon are of symmetric "look-ahead \& look-back" type, i.e., $\mathbb{N}_{i}=\{i-h, \cdots, i+h\} \cap$ $\{1, \cdots, N\} \backslash\{i\}$.

c) Information flow among followers is undirected, i.e., $j \in \mathbb{N}_{i} \Leftrightarrow i \in \mathbb{N}_{j}, i, j=1,2, \cdots, N$.

1.3) If $\tilde{G}$ satisfies the above Statements $\mathbf{1 . 1}$ and $\mathbf{1 . 2}$, platoon dynamics (17) are asymptotically stable if and only if

$$
\left\{\begin{array}{l}
k_{1}>0 \\
k_{2}>k_{1} \tau / \min _{i \in\{1,2, \cdots, N\}}\left(\lambda_{i} k_{3}+1\right) \\
k_{3}>-1 / \max _{i \in\{1,2, \cdots, N\}}\left(\lambda_{i}\right)
\end{array}\right.
$$
we have

Proof: From the definition of Laplacian matrix $L$ in (7),

$$
\left\{\begin{array}{l}
\sum_{j=1}^{N} l_{i j}=0 \\
l_{i i}=\sum_{j=1, j \neq i}^{N}\left|l_{i j}\right| \geq 0
\end{array} \quad, \quad i=1,2, \cdots, N .\right.
$$

Considering the definition of pinning matrix $P$, we have $p_{i} \geq 0$. Hence, for matrix $L+P$, we have

$$
\left|l_{i i}+p_{i}\right|=\left|l_{i i}\right|+\left|p_{i}\right| \geq \sum_{j=1, j \neq i}\left|l_{i j}\right|, i=1,2, \cdots, N
$$

By Lemma 1, all the eigenvalues of $L+P$ are located in the union of $N$ disks

$$
\bigcup_{i=1}^{N}\left\{\lambda \in \mathbb{C}|| \lambda-l_{i i}-p_{i}\left|\leq \sum_{j=1, j \neq i}^{N}\right| l_{i j} \mid\right\} .
$$

Then, the range of all the eigenvalues of $L+P$ lies in the disk $\left\{\lambda \in \mathbb{C}|| \lambda-\max _{i \in\{1,2, \cdots, N\}}\left(l_{i i}+p_{i}\right) \mid \leq \max _{i \in\{1,2, \cdots, N\}}\left(l_{i i}+p_{i}\right)\right\}$.

Hence, all the eigenvalues of $L+P$ lie within the union

$$
\{\lambda \in \mathbb{C} \mid \operatorname{Re}(\lambda)>0 \cup\{0\}\} .
$$

In addition, under the spanning tree assumption, there is at least one follower can obtain information from leader, i.e., $p_{i}=$ 1 , for at least one $i \in\{1,2, \ldots, N\}$. Without loss of generality, we assume there are two followers $r_{1}$ and $r_{2}$ pinned to leader, i.e., $p_{r_{1}}=1$ and $p_{r_{2}}=1$. Then, inequality (23) holds strictly for $i=r_{1}$ and $i=r_{2}$. We view $L+P$ as the matrix $Q$ defined in Lemma 2, then $J=\left\{r_{1}, r_{2}\right\}$ according to the definition in Lemma 2. Considering the spanning tree assumption, for any node $i$, which does not have a direct connection to the leader (i.e., $i \in J$ ), there must be a direct path $\left\{\alpha_{r}, \alpha_{i_{\zeta}}, \ldots, \alpha_{i_{1}}, \alpha_{i}\right\}$ connecting node $r \in J$ and node $i$, and the corresponding elements of $L+P$ (i.e., $l_{i i_{1}}+p_{i i_{1}}, l_{i_{1} i_{2}}+p_{i_{1} i_{2}}, \cdots, l_{i_{\zeta} r}+p_{i_{\zeta} r}$ ) are all nonzero. Therefore, $L+P$ is nonsingular according to 
Lemma 2, which implies that all the eigenvalues of $L+P$ are located in the open right-half plane by combining (26), i.e., $-\lambda_{i} \in \mathbb{C}_{-}, i=1,2, \ldots, N$.

To prove Statement 1.2, note that $L$ under assumption (a) is a lower triangular matrix, and $P$ is a diagonal matrix. Thus, $L+P$ is always a lower triangular matrix, which implies that all the eigenvalues of $L+P$ are real numbers, i.e.,

$$
\lambda_{i}=l_{i i}+p_{i}, \quad i=1,2, \cdots, N .
$$

Meanwhile, $L$ under assumption (b) or assumption (c) is a symmetric matrix, and hence $L+P$ is also symmetric, which implies that all the eigenvalues of $L+P$ are real numbers, i.e., $\lambda_{i} \in \mathbb{R}, i=1,2, \ldots, N$.

To prove Statement 1.3, we know that all the eigenvalues of $L+P$ are positive real numbers, by combining the results in the above two statements. According to Lemma 3, platoon dynamics (17) are asymptotically stable if and only if the real parts of the eigenvalues of matrices $A-\lambda_{i} B k^{T}, i=$ $1,2, \cdots, N$ are all negative. The characteristic polynomial of matrix $A-\lambda_{i} B k^{T}$ is

$$
\left|s I-\left(A-\lambda_{i} B k^{T}\right)\right|=s^{3}+\frac{\lambda_{i} k_{3}+1}{\tau} s^{2}+\frac{\lambda_{i} k_{2}}{\tau} s+\frac{\lambda_{i} k_{1}}{\tau} .
$$

The stability of (28) is examined using the Routh-Hurwitz stability criterion, shown in

$$
\begin{array}{ccc}
s^{3} & 1 & \frac{\lambda_{i} k_{2}}{\tau} \\
s^{2} & \frac{\lambda_{i} k_{3}+1}{\tau} & \frac{\lambda_{i} k_{1}}{\tau} \\
s^{1} & \frac{\lambda_{i} k_{2}\left(\lambda_{i} k_{3}+1\right)-\lambda_{i} k_{1} \tau}{\tau\left(\lambda_{i} k_{3}+1\right)} & \\
s^{0} & \frac{\lambda_{i} k_{1}}{\tau} . &
\end{array}
$$

Given the facts $\tau>0, \lambda_{i}>0, i=1,2, \ldots, N$, we have that (28) is asymptotically stable if and only if

$$
\left\{\begin{array}{l}
k_{1}>0 \\
k_{2}>k_{1} \tau /\left(\lambda_{i} k_{3}+1\right), \quad i=1,2, \cdots, N . \\
k_{3}>-1 / \lambda_{i}
\end{array}\right.
$$

Thus, $A-\lambda_{i} B k^{T}, i=1,2, \cdots, N$ are asymptotically stable, i.e., platoon dynamics (17) are asymptotically stable if and only if (21) are satisfied.

Remark 1: For Statement 1.1, similar results were established in [30], [41], and [42]. The proof in [41] relies on the fact that $L+P$ is irreducible when graph $\tilde{G}$ contains a spanning tree. The technique used in this paper is similar to [42].

Remark 2: In a platoon, if the vehicle acceleration is inaccessible, i.e., $k_{3}=0$, then the platoon dynamics (17) are asymptotically stable if and only if

$$
k_{1}>0, k_{2}>k_{1} \tau \text {. }
$$

Earlier development of platoons is radar-based, which lacks acceleration information of other vehicles. In such cases, as long as $k_{1}, k_{2}$ satisfy (31), the internal stability of platoon can be guaranteed.

Remark 3: The conclusion (31) is consistent with [21], [22]. In [21] and [22], similar results were obtained using partial differential equation approximation, but only suitable for platoons with BD and BDL topologies. The proof here extends their results, and is suitable for a large class of information flow topologies as long as they satisfy the conditions in Statements $\mathbf{1 . 1}$ and 1.2. These conditions can cover all aforementioned topologies in Fig. 1.

Remark 4: The conditions in Statements 1.1 and 1.2 guarantee the eigenvalues of corresponding matrix $L+P$ to be real numbers, which leads to a third-order Routh-Hurwitz stability criterion (29). Thus, the stabilizing thresholds of the linear control gains can be explicitly derived. For any given topology, the eigenvalues of $L+P$ may have imaginary part. Then, the Routh-Hurwitz stability criterion is sixth order, which is hard to explicitly derive the stabilizing thresholds.

Remark 5: The influence of graph $\tilde{G}$ on the stabilizing range of distributed controller gains is reflected by the eigenvalues of $L+P$. We can obtain a larger range of stabilizing controller gains by choosing the topology (i.e., with larger $\lambda_{\min }$ since $k_{3}$ is usually chose to be nonnegative number). The freedom from the additional stabilizing range could then be used to improve other performance indexes, e.g., string stability.

\section{B. Scalability Analysis for Typical Information Flow Topologies}

Theorem 1 explicitly establishes the stabilizing thresholds of linear controller gains for platoons under a large class of information flow topologies. In general, platoon reduces its stability margin with increasing number of vehicles [25], [31], [35]. Note that the stability margin is measured by the absolute value of the real part of the least stable closed-loop eigenvalue. In this section, by using matrix eigenvalue analysis, we will mathematically prove how the stability margin changes with platoon scale (i.e., scalability) under two typical topologies, i.e., BD topology and BDL topology. Before presenting the second theorem, we need the following Lemmas.

Lemma 4. [39]: Suppose that

$$
\widetilde{D}=\left[\begin{array}{cc}
D & y \\
y^{T} & d_{n}
\end{array}\right] \in \mathbb{R}^{n \times n}\left(\text { or } \widetilde{D}=\left[\begin{array}{cc}
d_{1} & y \\
y^{T} & D
\end{array}\right] \in \mathbb{R}^{n \times n}\right)
$$

is a symmetric matrix and $D$ is a (n-1)-by-(n-1) symmetric matrix. Let $\gamma_{1} \leq \gamma_{2} \leq \cdots \leq \gamma_{n}$ be the eigenvalues of $\tilde{D}$ and $\beta_{1} \leq \beta_{2} \leq \cdots \leq \beta_{n-1}$ be the eigenvalues of $D$. Then

$$
\begin{aligned}
\gamma_{1} \leq \beta_{2} \leq \cdots \leq \beta_{i-1} \leq & \gamma_{i} \leq \beta_{i+1} \\
& \leq \gamma_{i+1} \leq \cdots \leq \beta_{n-1} \leq \gamma_{n} .
\end{aligned}
$$

Lemma 5: Given a real polynomial

$$
p(s, \lambda)=s^{3}+\frac{\lambda k_{3}+1}{\tau} s^{2}+\lambda \frac{k_{2}}{\tau} s+\lambda \frac{k_{1}}{\tau}
$$

where $s$ denotes the independent variable, $k_{1}, k_{2}, k_{3}, \tau$ are nonzero constant real numbers, and parameter $\lambda \in \mathbb{R}$. If (33) is asymptotically stable, then the following statements hold:

5.1) Equation (33) has one characteristic root approaching $-1 / \tau$ and two characteristic roots approaching zero as $0(\lambda)$ when $\lambda$ goes to zero. 
5.2) Equation (33) has no characteristic root close to zero unless $\lambda$ is close to zero.

Proof: To prove Statement 5.1, consider a generic cubic polynomial:

$$
p(s)=s^{3}+b s^{2}+c s+d .
$$

When $\lambda$ goes to zero, we have

$$
\left\{\begin{array}{l}
b=\frac{\lambda k_{3}+1}{\tau}=\frac{1}{\tau}+O(\lambda) \\
c=\lambda \frac{k_{2}}{\tau}=O(\lambda) \\
d=\lambda \frac{k_{1}}{\tau}=O(\lambda)
\end{array} .\right.
$$

Note that (33) is stable if and only if $b, c, d,>0, b c>d$ according to the Routh-Hurwitz stability criterion. From the discriminant of cubic polynomial in [43], we have

$$
\Delta=b^{2} c^{2}+18 b c d-4 b^{3} d-4 c^{3}-27 d^{2} .
$$

Note the facts in (35), when the $\lambda$ is sufficient small, we have

$$
\Delta=O\left(\lambda^{2}\right)+O\left(\lambda^{2}\right)-O\left(\lambda^{1}\right)-O\left(\lambda^{2}\right)-O\left(\lambda^{3}\right)<0 .
$$

Hence, the polynomial (33) has one real characteristic root and one pair conjugate characteristic roots according to [43], when the $\lambda$ is sufficient small. Suppose the characteristic roots of (33) are $\xi, \gamma \pm j \beta$, where $j=\sqrt{-1}$, then we have

$$
p(s, \lambda)=s^{3}-(\xi+2 \gamma) s^{2}+\left(\gamma^{2}+\beta^{2}+2 \gamma \xi\right) s-\left(\gamma^{2}+\beta^{2}\right) \xi .
$$

According to (33) and (38), when the $\lambda$ is sufficient small, we have

$$
\left\{\begin{array}{l}
\xi+2 \gamma=-\frac{\lambda k_{3}+1}{\tau}=-\frac{1}{\tau}+O(\lambda) \\
\gamma^{2}+\beta^{2}+2 \gamma \xi=\lambda \frac{k_{2}}{\tau}=O(\lambda) \\
\left(\gamma^{2}+\beta^{2}\right) \xi=-\lambda \frac{k_{1}}{\tau}=O(\lambda)
\end{array} .\right.
$$

Hence, we have

$$
\left\{\begin{array}{l}
\xi=-\frac{1}{\tau}+O(\lambda) \\
\gamma^{2}+\beta^{2}=O(\lambda) \\
\gamma=0(\lambda)
\end{array} .\right.
$$

Therefore, if $\lambda$ goes to zero, then polynomial (33) has one characteristic root $\xi$ approaching $-1 / \tau$ and two characteristic roots $\gamma \pm j \beta$ approaching zero as the speed of $O(\lambda)$.

To prove Statement 5.2, suppose that the characteristic roots of (33) are $\xi_{1}, \xi_{2}, \xi_{3}$, we have

$$
\left\{\begin{array}{l}
\xi_{1}+\xi_{2}+\xi_{3}=-\frac{\lambda k_{3}+1}{\tau} \\
\xi_{1} \cdot \xi_{2}+\xi_{2} \cdot \xi_{3}+\xi_{1} \cdot \xi_{3}=\lambda \frac{k_{2}}{\tau} \\
\xi_{1} \cdot \xi_{2} \cdot \xi_{3}=-\lambda \frac{k_{1}}{\tau}
\end{array} .\right.
$$

Since $k_{1}, k_{2}, k_{3}, \tau$ are non-zero constant numbers, $\xi_{1}, \xi_{2}, \xi_{3}$ would not converge to zero unless $\lambda$ is close to zero.

Lemma 6: Given two matrices $D=\alpha I_{n}$ and $E \in \mathbb{R}^{n \times n}$. Then $\sigma_{i}(D+\beta E)=\alpha+\beta \sigma_{i}(E), i=1,2, \cdots, n$.

Proof: Take any eigenvalue $\sigma_{i}(E)$ and the corresponding eigenvector $w_{i} \mathbb{C}^{n}$. Then $(D+\beta E) w_{i}=D w_{i}+\beta E w_{i}=$ $\alpha w_{i}+\beta \sigma_{i}(E) w_{i}=\left(\alpha+\beta \sigma_{i}(E)\right) w_{i}$.
Lemma 7: Given any positive definite matrix $D \in \mathbb{R}^{n \times n}$. Then $D^{-1}$ is also positive definite and $\sigma_{n-i+1}\left(D^{-1}\right), i=$ $1,2, \cdots, n$.

Proof: Take any eigenvalue $\sigma_{i}(D)$ and the corresponding eigenvector $w_{i} \in \mathbb{C}^{n}$. Then $w_{i}=D^{-1} D w_{i}=D^{-1} \sigma_{i}(D) w_{i}$. Therefore, we have $D^{-1} w_{i}=1 / \sigma_{i}(D) w_{i}$.

Using lemmas stated above, we state the second theorem in this paper.

Theorem 2: Consider the homogeneous platoon using linear controllers with the closed-loop dynamics as in (17), and the controller satisfies the conditions in Statement 1.3 of Theorem 1. The following statements hold:

2.1) If graph $\tilde{G}$ is in BD topology, then the stability margin of platoon decays to zero as $O\left(1 / N^{2}\right)$.

2.2) If graph $\tilde{G}$ is in BDL topology, then the eigenvalues of matrix $L_{\mathrm{BDL}}+P_{\mathrm{BDL}}$ are bigger or equal to one. Moreover, only $\sigma_{\min }\left(L_{\mathrm{BDL}}+P_{\mathrm{BDL}}\right)=1$, and $\sigma_{2}\left(L_{\mathrm{BDL}}+P_{\mathrm{BDL}}\right)$ decays to one as $O\left(1 / N^{2}\right)$.

Proof of Statement 2.1: Under the BD topology, $L$ and $P$ are noted as $L_{\mathrm{BD}} \in \mathbb{R}^{N \times N}$ and $P_{\mathrm{BD}} \in \mathbb{R}^{N \times N}$, expressed as:

$$
L_{\mathrm{BD}}=\left[\begin{array}{cccccccc}
1 & -1 & & & & & \\
-1 & 2 & -1 & & & \\
& -1 & 2 & \ddots & & \\
& & -1 & 2 & \ddots & \\
& & & \ddots & \ddots & -1 \\
& & & & & -1 & 1
\end{array}\right]
$$

and

$$
P_{\mathrm{BD}}=\left[\begin{array}{cccc}
1 & & & \\
& 0 & & \\
& 0 & & \\
& & \ddots & \\
& & & 0
\end{array}\right] .
$$

The sum of $L_{\mathrm{BD}}$ and $P_{\mathrm{BD}}$ can be rewritten as

$$
L_{\mathrm{BD}}+P_{\mathrm{BD}}=\left[\begin{array}{cc}
Z_{\mathrm{BD}} & -e_{\mathrm{BD}} \\
-e_{\mathrm{BD}}^{T} & 1
\end{array}\right],
$$

where $Z_{\mathrm{BD}} \in \mathbb{R}^{(N-1) \times(N-1)}, e_{\mathrm{BD}} \in \mathbb{R}^{(N-1) \times 1}$ defined as

$$
Z_{\mathrm{BD}}=\left[\begin{array}{ccccc}
2 & -1 & & & \\
-1 & 2 & -1 & & \\
& -1 & 2 & \ddots \\
& & \ddots & \ddots & -1 \\
& & & -1 & 2
\end{array}\right], \quad e_{\mathrm{BD}}=\left[\begin{array}{c}
0 \\
0 \\
\vdots \\
1
\end{array}\right] \text {. }
$$

Further, $Z_{\mathrm{BD}}$ is decomposed to $Z_{B D}=2 I_{N-1}-W$, where $W \in \mathbb{R}^{(N-1) \times(N-1)}$ is defined as

$$
W=\left[\begin{array}{ccccc}
0 & 1 & & & \\
1 & 0 & 1 & & \\
& 1 & 0 & \ddots & \\
& & \ddots & \ddots & 1 \\
& & & 1 & 0
\end{array}\right] .
$$


It is known from [44] that the eigenvalues of matrix $W$ are

$$
\sigma_{N-i}(W)=2 \cos \frac{i \pi}{N} .
$$

According to Lemma 6, the eigenvalues of matrix $Z_{\mathrm{BD}}$ are

$$
\sigma_{i}\left(Z_{\mathrm{BD}}\right)=2-2 \cos \frac{i \pi}{N}=4 \sin ^{2} \frac{i \pi}{2 N} .
$$

By Lemma 4, we have

$$
\left\{\begin{array}{l}
\sigma_{\min }\left(L_{\mathrm{BD}}+P_{\mathrm{BD}}\right) \leq \sigma_{\min }\left(Z_{\mathrm{BD}}\right)=4 \sin ^{2} \frac{i \pi}{2 N} \leq \frac{\pi^{2}}{N^{2}} \\
\sigma_{2}\left(L_{\mathrm{BD}}+P_{\mathrm{BD}}\right) \leq \sigma\left(Z_{\mathrm{BD}}\right)=4 \sin ^{2} \frac{\pi}{N} \leq \frac{4 \pi^{2}}{N^{2}}
\end{array}\right.
$$

Equation (44) actually gives the upper bounds of $\sigma_{\min }\left(L_{\mathrm{BD}}+\right.$ $\left.P_{\mathrm{BD}}\right)$ and $\sigma_{2}\left(L_{\mathrm{BD}}+P_{\mathrm{BD}}\right)$.

In addition, according to Statements $\mathbf{1 . 1}$ and $\mathbf{1 . 2}, L_{\mathrm{BD}}+$ $P_{\mathrm{BD}}$ is symmetric positive definite. Hence, from Lemma 7 we know that $\sigma_{i}\left(\left(L_{\mathrm{BD}}+P_{\mathrm{BD}}\right)^{-1}\right)>0$ and $\sigma_{\min }\left(L_{\mathrm{BD}}+P_{\mathrm{BD}}\right)=$ $1 / \sigma_{\max }\left(\left(L_{\mathrm{BD}}+P_{\mathrm{BD}}\right)^{-1}\right)$. As proved in [11], $\left(L_{\mathrm{BD}}+P_{\mathrm{BD}}\right)^{-1}$ is equal to

$$
\left(L_{\mathrm{BD}}+P_{\mathrm{BD}}\right)^{-1}=\left[\begin{array}{ccccc}
1 & 1 & \cdots & 1 & 1 \\
1 & 2 & \cdots & 2 & 2 \\
\vdots & \vdots & \ddots & \vdots & \vdots \\
1 & 2 & \cdots & N-1 & N-1 \\
1 & 2 & \cdots & N-1 & N
\end{array}\right] .
$$

According to Lemma 1, we know

$$
\sigma_{\max }\left(\left(L_{\mathrm{BD}}+P_{\mathrm{BD}}\right)^{-1}\right) \leq N+\sum_{k=1}^{N-1} k=\frac{N(N+1)}{2} .
$$

Therefore, we obtain the lower bound for the least eigenvalue of $L_{\mathrm{BD}}+P_{\mathrm{BD}}$, i.e.,

$$
\sigma_{\min }\left(L_{\mathrm{BD}}+P_{\mathrm{BD}}\right) \geq \frac{2}{N(N+1)} \geq \frac{1}{N^{2}} .
$$

Based on (44) and (45), we can claim that $\sigma_{\min }\left(L_{\mathrm{BD}}+P_{\mathrm{BD}}\right)$ approaches zero as $O\left(1 / N^{2}\right)$. According to Lemma 3 and Lemma 5, we can claim that the stability of platoon, which is measured by the real part of the least stable closed-loop eigenvalue, decays to zero as $O\left(\sigma_{\min }\left(L_{\mathrm{BD}}+P_{\mathrm{BD}}\right)\right) O\left(1 / N^{2}\right)$, where $N$ is the number of following vehicles, if the information flow is in BD topology.

Proof of Statement 2.2: Under the BDL topology, it is assumed that the platoon has $N+1$ following vehicles with the purpose of reusing some properties in Statement 2.1. The matrices $L$ and $P$ are noted as $L_{\mathrm{BDL}} \in \mathbb{R}^{(N+1) \times(N+1)}$ and $P_{\mathrm{BDL}} \in \mathbb{R}^{(N+1) \times(N+1)}$, the sum of which is expressed as

$$
L_{\mathrm{BDL}}+P_{\mathrm{BDL}}=\left[\begin{array}{ccccc}
2 & -1 & & & \\
-1 & 3 & -1 & & \\
& -1 & 3 & \ddots & \\
& & \ddots & \ddots & -1 \\
& & & -1 & 2
\end{array}\right] .
$$

Further, $L_{\mathrm{BDL}}+P_{\mathrm{BDL}}$ is decomposed to $L_{\mathrm{BDL}}+P_{\mathrm{BDL}}=$ $I_{N+1}+Z_{\mathrm{BDL}}$, where $Z_{\mathrm{BDL}} \in \mathbb{R}^{(N+1) \times(N+1)}$ is defined as

$$
Z_{\mathrm{BDL}}=\left[\begin{array}{ll}
1 & * \\
* L_{\mathrm{BD}} & +P_{\mathrm{BD}}
\end{array}\right] .
$$

According to Lemma 4, we have $\sigma_{\min }\left(Z_{\mathrm{BDL}}\right) \leq \sigma_{\min }\left(L_{\mathrm{BD}}+\right.$ $\left.P_{\mathrm{BD}}\right) \leq \sigma_{2}\left(Z_{\mathrm{BDL}}\right) \leq \sigma_{2}\left(L_{\mathrm{BD}}+P_{\mathrm{BD}}\right)$. Therefore, we get the bounds for $\sigma_{\min }\left(L_{\mathrm{BDL}}+P_{\mathrm{BDL}}\right)$ and $\sigma_{2}\left(L_{\mathrm{BDL}}+P_{\mathrm{BDL}}\right)$ based on Lemma 6:

$$
\left\{\begin{array}{l}
\sigma_{\min }\left(L_{\mathrm{BDL}}+P_{\mathrm{BDL}}\right) \leq 1+\sigma_{\min }\left(L_{\mathrm{BD}}+P_{\mathrm{BD}}\right) \leq 1+\frac{\pi^{2}}{N^{2}} \\
\sigma_{2}\left(L_{\mathrm{BDL}}+P_{\mathrm{BDL}}\right) \leq 1+\sigma_{2}\left(L_{\mathrm{BD}}+P_{\mathrm{BD}}\right) \leq 1+\frac{4 \pi^{2}}{N^{2}} \\
\sigma_{2}\left(L_{\mathrm{BDL}}+P_{\mathrm{BDL}}\right) \geq 1+\sigma_{\min }\left(L_{\mathrm{BD}}+P_{\mathrm{BD}}\right) \geq 1+\frac{1}{N^{2}}
\end{array} .\right.
$$

Define vector $1=[1,1, \ldots, 1]^{T} \in \mathbb{R}^{N+1}$, then it is easy to know from (46) that

$$
\left(L_{\mathrm{BDL}}+P_{\mathrm{BDL}}\right) \cdot \mathbf{1}=\mathbf{1} .
$$

Hence, we have $\sigma_{\min }\left(L_{\mathrm{BDL}}+P_{\mathrm{BDL}}\right)=1$. According to (47), we also have $\sigma_{2}\left(L_{\mathrm{BDL}}+P_{\mathrm{BDL}}\right)=1+O\left(1 /(N+1)^{2}\right)$.

Remark 6: According to Lemma 3 and Lemma 5, given any information flow topology satisfying the conditions in Statements 1.1 and $\mathbf{1 . 2}$ and the linear controller gains satisfying Statement 1.3, then:

1) The platoon dynamics (17) will not have eigenvalues close to zero unless $\sigma_{\min }(L+P)$ is close to zero, i.e., the closed-loop system have certain stability margin.

2) The stability margin of platoon dynamics (17) decays to zero as $O\left(\sigma_{\min }(L+P)\right)$, if $\sigma_{\min }(L+P)$ approaches zero with increasing platoon size.

Remark 7: In theory, the feasibility to handle the scalability of platoons depends on whether the eigenvalues of $L+P$ are analytically obtainable. In general, to analytically obtain these matrix eigenvalues is rather difficult. Up to now, the authors can only analyze the matrices related to BD and BDL topologies, which also received extensive research interest in today's literature, e.g., [21], [25], [31], [32].

Remark 8: Statement 2.1 demonstrates the real part of least stable eigenvalue decays to zero as $O\left(1 / N^{2}\right)$ for platoons under $\mathrm{BD}$ topology, which is independent of the design of identical controllers. This conclusion conforms to [25], [31] and [32], in which partial differential equation is used to approximate the platoon dynamics. In addition, our research extends their results by taking into account the inertial delay of vehicle longitudinal dynamics, whereas that of each vehicle in [25], [31] and [32] is assumed as an ideal double integrator. Thus, our consideration is able to provide more accurate prediction to the scalability limitation in real-world implementation.

Remark 9: Statement 2.2 is an extension of Statement 2.1 when broadcasting the leader information. The least eigenvalue of matrix $L_{\mathrm{BDL}}+P_{\mathrm{BDL}}$ for BDL topology is always equal to one. Considering Lemma 3 and Lemma 5, it is easy to know that there is a constant gap (stability margin) between the least stable closed-loop eigenvalue and the imaginary axis, which is independent of the platoon scale $N$. Hence, we claim that the 
TABLE I

EIGENVALUES FOR DIFFERENT INFORMATION FLOW TOPOLOGIES IN Fig. $1(N=10)$

\begin{tabular}{cccccc}
\hline a) PF & (b) PLF & (c) BD & (d) BDL & (e)TPF & (f)TPLF \\
\hline 1 & 1 & 0.0223 & 1.0000 & 1 & 1 \\
1 & 2 & 0.1981 & 1.0979 & 2 & 2 \\
1 & 2 & 0.5339 & 1.3820 & 2 & 3 \\
1 & 2 & 1.0000 & 1.8244 & 2 & 3 \\
1 & 2 & 1.5550 & 2.3820 & 2 & 3 \\
1 & 2 & 2.1495 & 3.0000 & 2 & 3 \\
1 & 2 & 2.7307 & 3.6180 & 2 & 3 \\
1 & 2 & 3.2470 & 4.1756 & 2 & 3 \\
1 & 2 & 3.6525 & 4.6180 & 2 & 3 \\
1 & 2 & 3.9111 & 4.9021 & 2 & 3 \\
\hline
\end{tabular}

scalability of platoons under BDL topology can be guaranteed when the controller gains are properly designed.

Remark 10: For the undirected topology $\tilde{G}$ with all the followers pinned to the leader, the corresponding pinning matrix $P$ is an identity matrix, i.e., $P=I_{N}$, and $L$ is a symmetric matrix. According to Lemma 1, we know that $\sigma_{i}(L) \geq 0$ and $\sigma_{i}(L+P)=1+\sigma_{i}(L) \geq 1, i=1,2, \cdots, N$. Hence, the property stated in Remark 9 is also correct for such kind of topology, i.e., there exists certain size-independent stability margin.

Remark 11: Using the similar analysis framework in Theorem 2, it's easy to know that all the eigenvalues of associated matrix $L_{\mathrm{PF}}+P_{\mathrm{PF}}$ for $\mathrm{PF}$ topology are equal to one, which is also independent of the platoon size $N$. It seems that platoon under PF topology will have good scalability performance. However, the algebraic multiplicity of the least stable eigenvalue for PF topology is as same as the platoon size $N$, which leads to algebraic growth (peaking phenomena) in the transient process. In this case, there are motion modes as $t^{k} e^{\operatorname{Re}(\mu) t}, k=1, \cdots, N-1$, where $\mu$ is the stable closed-loop eigenvalue with $N$-th algebraic multiplicity.

\section{Simulation Results and Discussions}

Numerical simulations are conducted to illustrate the main results. We consider a homogeneous platoon with 11 identical vehicles ( 1 leader and 10 followers) interconnected by the six information flow topologies shown in Fig. 1. The acceleration or deceleration of the leader can be viewed as disturbances in a platoon. The initial state of the leader is set as $s_{0}(t)=0, v_{0}=$ $20 \mathrm{~m} / \mathrm{s}$ and the desired trajectory is given by

$$
v_{0}= \begin{cases}20 \mathrm{~m} / \mathrm{s} & t \leq 5 \mathrm{~s} \\ 20+2 t \mathrm{~m} / \mathrm{s} & 5 s<t \leq 10 \mathrm{~s} \\ 30 \mathrm{~m} / \mathrm{s} & t>10 \mathrm{~s} .\end{cases}
$$

The eigenvalues of associated matrix $L+P$ for these six information flow topologies are listed in Table I All the eigenvalues are positive real numbers, which are consistent with

\section{Statements 1.1 and 1.2.}

In the simulations, the desired spacing is set as $d_{i-1, i}=20 \mathrm{~m}$ and the vehicle length is equal to $4 \mathrm{~m}$. The initial state of the platoon is set as the desired state, i.e., the initial spacing errors and velocity errors are all equal to 0. Two scenarios, i.e., stability and instability, have been simulated by considering two groups of specific parameters (see Table II). Fig. 2 demonstrates spacing errors for different information flow topologies

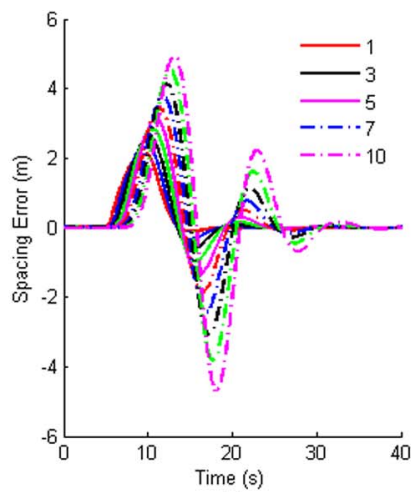

(a)

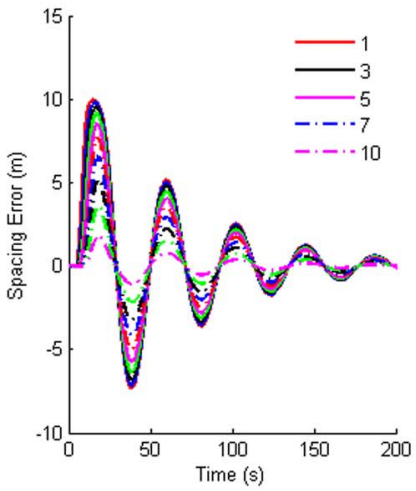

(c)

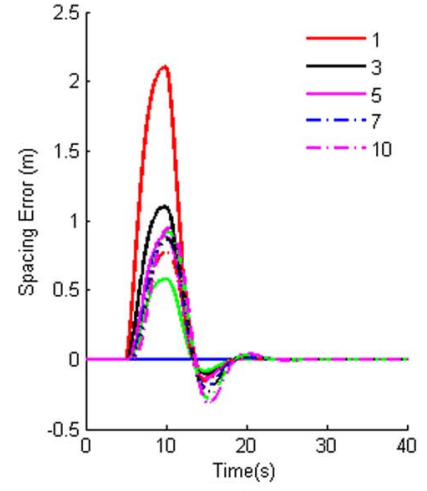

(e)

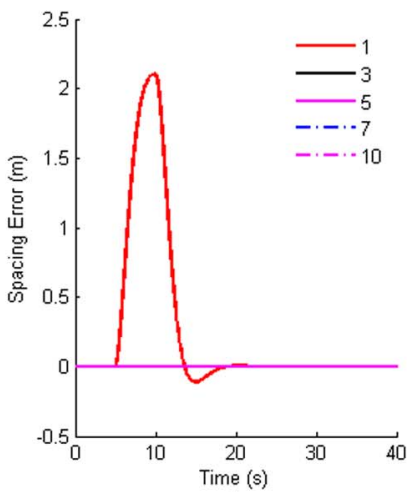

(b)

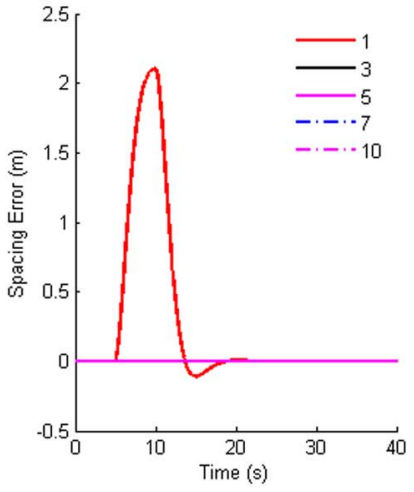

(d)

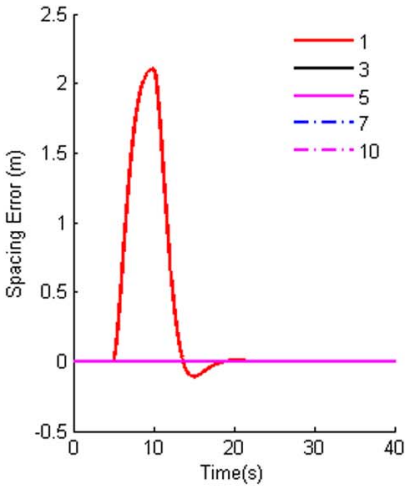

(f)
Fig. 2. Performance of stable platoon when Theorem 1.3 is satisfied. (a): PF. (b): PLF. (c):BD. (d):BDL. (e): TPF. (f): TPLF.

TABLE II

PARAMETERS FOR THE PlATOON

\begin{tabular}{ccc}
\hline Parameters & Scenario 1 & Scenario 2 \\
\hline$\tau$ & 0.5 & 0.5 \\
$k_{1}$ & 1 & 1 \\
$k_{2}$ & 2 & 0.2 \\
$k_{3}$ & 1 & 1 \\
Theorem 1.3 & Satisfied & Dissatisfied \\
\hline
\end{tabular}

(i.e., Fig. 1(a)-(f)) in Scenario 1, whose parameters are listed in Table II. As the parameters in Scenario 1 satisfy the internal stability condition (21), i.e., Statement $\mathbf{1 . 3}$ holds, the motion of the vehicles is stable for all information topologies. It is noted that for the spacing errors under PLF, BDL and TPLF topologies shown in Fig. 2, only the first follower has nonzero spacing error and other followers has almost zero spacing 


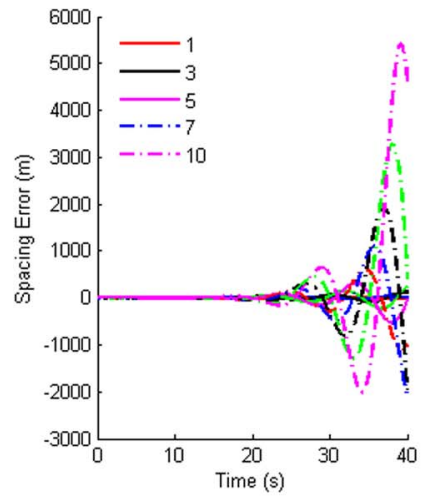

(a)

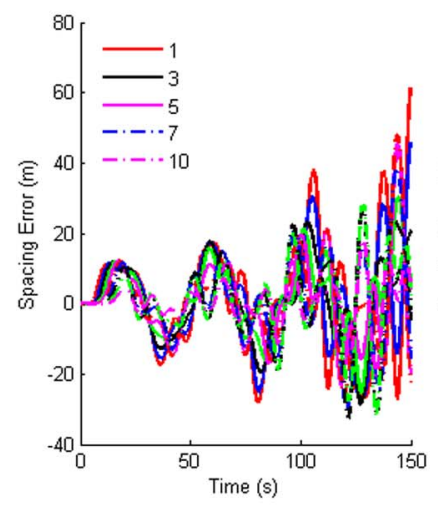

(c)

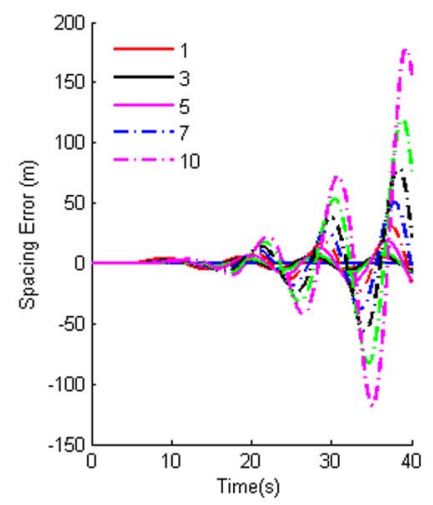

(e)

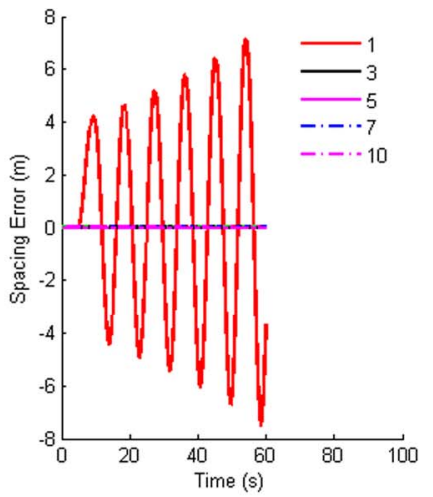

(b)

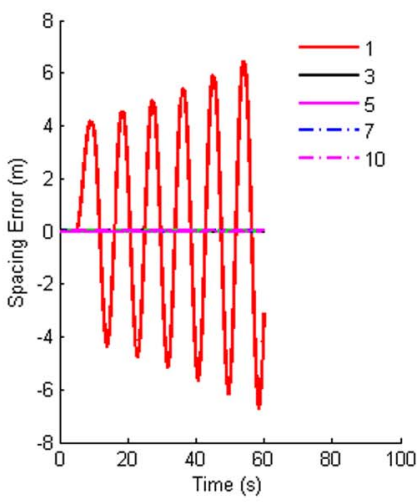

(d)

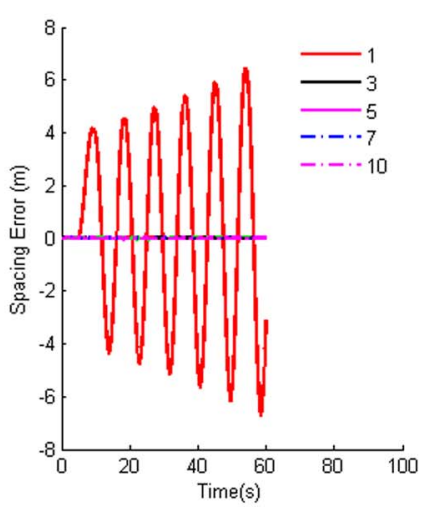

(f)
Fig. 3. Performance of unstable platoon when Theorem 1.3 is dissatisfied. (a): PF. (b): PLF. (c): BD. (d): BDL. (e): TPF. (f): TPLF.

errors. The reason is that all the followers in the platoon are pinned to the leader and have zero initial errors. Hence, the followers have similar dynamic evolution, which means the spacing errors between adjacent vehicles are close to zero. On the other hand, the parameters in Scenario 2 do not satisfy the stability condition (21), so instability occurs. Considering this fact, Fig. 3 shows the instability motions of the platoon.

To verify Lemma 5, the three characteristic roots of (33) have been numerically calculated, shown in Fig. 4. We can clearly observe that in this group of parameters, i.e., $k_{1}=1$, $k_{2}=2, k_{3}=1, \tau=0.5$ one real characteristic root approaches $-1 / \tau$ and the real part of other two conjugate characteristic roots approach zero as $O(\lambda)$ when $\lambda$ approaches zero. To verify the first statement in Theorem 2, we have calculated $\sigma_{\min }\left(L_{\mathrm{BDL}}+P_{\mathrm{BDL}}\right)$ for $\mathrm{BD}$ topology and its upper/lower
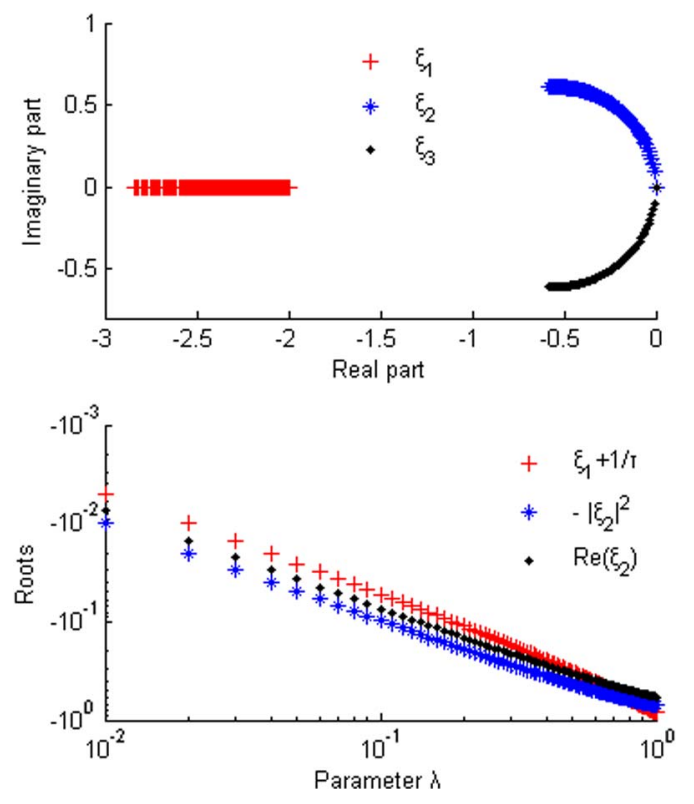

Fig. 4. Three characteristic roots of (33), where $k_{1}=1, k_{2}=2, k_{3}=1$, $\tau=0.5$.

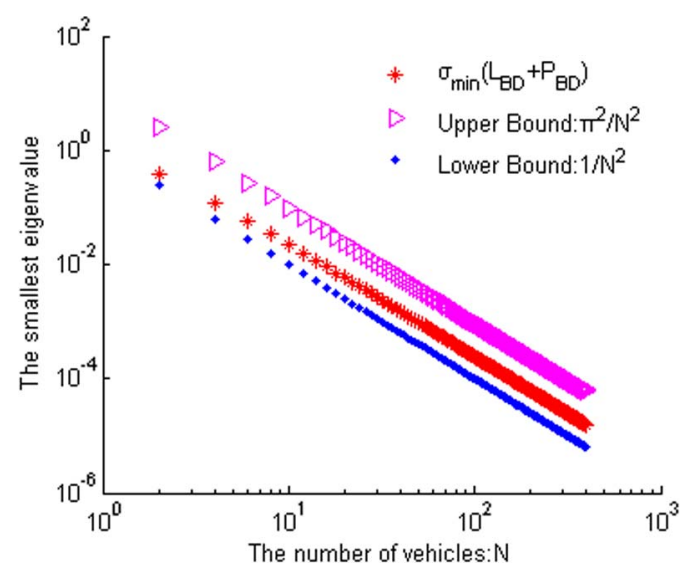

Fig. 5. $\sigma_{\min }\left(L_{\mathrm{BD}}+P_{\mathrm{BD}}\right)$ for $\mathrm{BD}$ topology.

bound under different platoon scale, which is shown in Fig. 5. We can observe that the upper/lower bounds given in Theorem 2 are quite accurate. Fig. 6 shows the $\sigma_{\min }\left(L_{\mathrm{BDL}}+P_{\mathrm{BDL}}\right)$ and $\sigma_{2}\left(L_{\mathrm{BDL}}+P_{\mathrm{BDL}}\right)$ for BDL topology under different sizes of platoon, which obviously confirms with the second statement in Theorem 2.

\section{CONCLUSiON}

This paper studies the influence of information flow topology on the internal stability and scalability of homogeneous vehicular platoons moving in a rigid formation. Using the exact feedback linearization, a linearized vehicle longitudinal dynamic model is derived which takes into account the inertial delay of powertrain dynamics. Directed graph topologies are employed to model allowable information flow among vehicles, including both radar-based and communication-based. Linear distributed controllers are designed, leading to platoon 


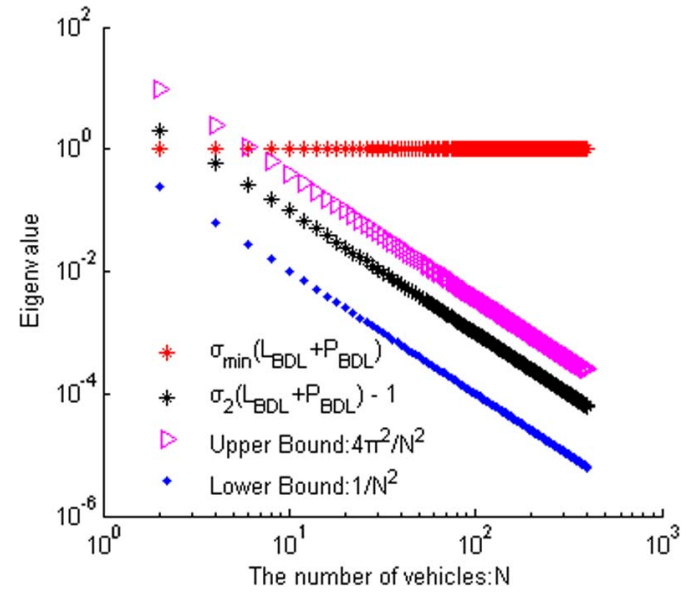

Fig. 6. $\sigma_{\mathrm{min}}\left(L_{\mathrm{BDL}}+P_{\mathrm{BDL}}\right)$ and $\sigma_{2}\left(L_{\mathrm{BDL}}+P_{\mathrm{BDL}}\right)$ for BDL topology.

closed-loop dynamics under the constant distance policy. The main conclusions are:

1) The first main theorem explicitly establishes the stabilizing thresholds of linear decentralized controller gains for platoons, which is suitable for a large class of information flow topologies. This unified internal stability theorem is derived though using the algebraic graph theory and Routh-Hurwitz stability criterion.

2) The second main theorem shows that the real part of the least stable closed-loop eigenvalue decays to zero as $O\left(1 / N^{2}\right)$ under bidirectional topology. Hence, such topology with identical linear controller suffers from fundamental limitations on the scalability of homogenous vehicle platoon. If the leader information is broadcasted to every followers, resulting in the socalled bidirectional-leader topology, the scalability of platoon can be significantly improved, because the stability margin is always bounded away from zero and independent of the platoon size.

Unsolved topics for future research include the unified internal stability and scalability theorem for heterogeneous platoons with non-identical controllers. In addition, there is a need to address the string stability issue for platoon under different information topologies.

\section{ACKNOWLEDGMENT}

The authors would like to thank Prof. Le-Yi Wang of Wayne State University for his constructive suggestions on information flow topology design and vehicle dynamics simplification.

\section{REFERENCES}

[1] F. Y. Wang, "Parallel control and management for intelligent transportation systems: Concepts, architectures, and applications," IEEE Trans. Intell. Transp. Syst., vol. 11, no. 3, pp. 630-638, Sep. 2011.

[2] R. Horowitz and P. Varaiya, "Control design of an automated highway system," Proc. IEEE, vol. 88, no. 7, pp. 913-925, Jul. 2000.

[3] J. Zhang et al., "Data-driven intelligent transportation systems: A survey," IEEE Trans. Intell. Transp. Syst., vol. 12, no. 4, pp. 1624-1639, Dec. 2011.

[4] W. Levine and M. Athans, "On the optimal error regulation of a string of moving vehicles," IEEE Trans. Autom. Control, vol. AC-11, no. 3, pp. 355-361, Jul. 1966.

[5] S. Shladover, "Review of the state of development of advanced vehicle control systems (AVCS)," Veh. Syst. Dyn., no. 6/7, pp. 551-595, Jul. 1995.
[6] S. Darbha and J. K. Hedrick, "Constant spacing strategies for platooning in automated highway systems," Trans. ASME, J. Dyn. Syst. Meas. Control, vol. 121, no. 3, pp. 462-470, Sep. 1999.

[7] S. Stankovic, M. Stanojevic, and D. Siljak, "Decentralized overlapping control of a platoon of vehicles," IEEE Trans. Control Syst. Technol., vol. 8 , no. 5, pp. 816-832, Sep. 2000.

[8] S. Kato, S. Tsugawa, K. Tokuda, T. Matsui, and H. Fujii, "Vehicle control algorithms for cooperative driving with automated vehicles and intervehicle communications," IEEE Trans. Intell. Transp. Syst., vol. 3, no. 3, pp. 155-161, Sep. 2002.

[9] P. Seiler, A. Pant, and J. Hedrick, "Disturbance propagation in vehicle strings," IEEE Trans. Autom. Control, vol. 49, no. 10, pp. 1835-1842, Oct. 2004.

[10] G. Naus, R. Vugts, J. Ploeg, M. Molengraft, and M. Steinbuch, "String-stable CACC design and experimental validation: A frequencydomain approach," IEEE Trans. Veh. Technol., vol. 59, pp. 4268-4279, Nov. 2010.

[11] P. Barooah and J. Hespanha, "Error amplification and disturbance propagation in vehicle strings with decentralized linear control," in Proc. IEEE Conf. Decision Control, 2005, pp. 4964-4969.

[12] S. Darbha and K. Rajagopal, "Information flow and its relation to the stability of the motion of vehicles in a rigid formation," in Proc. Amer. Control Conf., 2005, pp. 1853-1858.

[13] P. Kavathekar and Y. Chen, "Draft: Vehicle platooning: A brief survey and categorization," in Proc. ASME Int. Des. Eng. Tech. Conf. Comput. Inf. Eng. Conf., 2011, pp. 829-845.

[14] Y. Zhang, E. Kosmatopoulos, P. Ioannou, and C. Chien, "Using front and back information for tight vehicle following maneuvers," IEEE Trans. Veh. Technol., vol. 48, no. 1, pp. 319-328, Jan. 1999.

[15] R. Rajamani, H. Tan, B. Law, and W. Zhang, "Demonstration of integrated longitudinal and lateral control for the operation of automated vehicles in platoons," IEEE Trans. Control Syst. Technol., vol. 8, no. 4, pp. 695-708, Jul. 2000.

[16] M. Khatir and E. Davidson, "Bounded stability and eventual string stability of a large platoon of vehicles using non-identical controllers," in Proc. IEEE Conf. Decision Control, 2004, pp. 1111-1116.

[17] J. Ploeg, N. van de Wouw, and H. Nijmeijer "Lp string stability of cascaded systems: Application to vehicle platooning," IEEE Trans. Control Syst. Technol., vol. 22, no. 2, pp. 786-793, Mar. 2013.

[18] J. Ploeg, D. P. Shukla, N. van de Wouw, and H. Nijmeijer, "Controller synthesis for string stability of vehicle platoons," IEEE Trans. Intell. Transp. Syst., vol. 15, no. 2, pp. 854-865, Apr. 2014.

[19] D. Swaroop, J. K. Hedrick, C. C. Chien, and P. Ioannou, "A comparison of spacing and headway control laws for automatically controlled vehicles," Veh. Syst. Dyn., vol. 23, no. 1, pp. 597-625, Jan. 1994.

[20] L. Xiao and F. Gao, "Practical string stability of platoon of adaptive cruise control vehicles," IEEE Trans. Intell. Transp. Syst., vol. 12, no. 4, pp. 1184-1194, Dec. 2011.

[21] A. Ghasemi, R. Kazemi, and S. Azadi, "Stable decentralized control of platoon of vehicles with heterogeneous information feedback," IEEE Trans. Veh. Technol., vol. 62, no. 9, pp. 4299-4308, Nov. 2013.

[22] A. Ghasemi, R. Kazemi, and S. Azadi, "Stability analysis of bidirectional adaptive cruise control with asymmetric information flow," Proc. Inst. Mech. Eng. C, J. Mech. Eng. Sci., vol. 229, no. 2, pp. 216-226, 2015.

[23] S. Oncu, J. Ploeg, N. van de Wouw, and H. Nijmeijer, "Cooperative adaptive cruise control: Network-aware analysis of string stability," IEEE Trans. Intell. Transp. Syst., vol. 15, no. 4, pp. 1527-1537, Aug. 2014.

[24] W. B. Dunbar and S. C. Derek "Distributed receding horizon control of vehicle platoons: Stability and string stability," IEEE Trans. Autom. Control, vol. 57, no. 3, pp. 620-633, Mar. 2012.

[25] H. Hao and P. Barooah, "Stability and robustness of large platoons of vehicles with double-integrator models and nearest neighbor interaction," Int. J. Robust Nonlinear Control, vol. 23, no. 18, pp. 2097-2122, Dec. 2013.

[26] F. Qu, F. Wang, and L. Yang, "Intelligent transportation spaces: Vehicles, traffic, communications, and beyond," IEEE Commun. Mag., vol. 48, no. 11, pp. 136-142, Nov. 2010.

[27] T. Willke, P. Tientrakool, and N. Maxemchuk, "A survey of inter-vehicle communication protocols and their applications," IEEE Commun. Surveys Tuts., vol. 11, no. 2, pp. 3-20, 2nd Quart. 2009.

[28] J. Ploeg, A. Serrarens, and G. Heijenk, "Connect \& Drive: Design and evaluation of cooperative adaptive cruise control for congestion reduction," J. Mod. Transp., vol. 19, no. 3, pp. 207-213, Sep. 2011.

[29] S. Darbha and P. Pagilla, "Limitations of employing undirected information flow graphs for the maintenance of rigid formations for heterogeneous vehicles,” Int. J. Eng. Sci., vol. 48, no. 11, pp. 1164-1178, Nov. 2010. 
[30] R. Olfati-Saber and R. Murray, "Consensus problems in networks of agents with switching topology and time-delays," IEEE Trans. Autom. Control, vol. 49, no. 9, pp. 1520-1533, Sep. 2004.

[31] H. Hao and P. Barooah, "Control of large 1D networks of double integrator agents: role of heterogeneity and asymmetry on stability margin," in Proc IEEE Conf. Decision Control, 2010, pp. 7395-7400.

[32] H. Hao, P. Barooah, and P. G. Mehta "Stability margin scaling laws of distributed formation control as a function of network structure," IEEE Trans. Autom. Control, vol. 56, no. 4, pp. 923-929, Apr. 2011.

[33] S. Li, K. Li, R. Rajamani, and J. Wang, "Model predictive multi-objective vehicular adaptive cruise control," IEEE Trans. Control Syst. Technol., vol. 19, no. 3, pp. 556-566, May 2011.

[34] S. L. Eben, K. Li, and J. Wang, "Economy-oriented vehicle adaptive cruise control with coordinating multiple objectives function," Veh. Syst. Dyn., vol. 51, no. 1, pp. 1-17, Jan. 2013.

[35] M. Jovanovic and B. Bamieh, "On the ill-posedness of certain vehicular platoon control problems," IEEE Trans. Autom. Control, vol. 50, no. 9, pp. 1307-1321, Sep. 2005.

[36] C. Godsil and G. Royle, Algebraic Graph Theory. New York, NY, USA: Springer-Verlag, 2001

[37] X. Wang and G. Chen, "Pinning control of scale-free dynamical networks," Phys. A, Stat. Mech. Appl., vol. 310, no. 3/4, pp. 521-531, Jul. 2002.

[38] W. Ren and R. Beard, "Consensus seeking in multiagent systems under dynamically changing interaction topologies," IEEE Trans. Autom. Control, vol. 50, no. 5, pp. 655-661, May 2005.

[39] R. Horn and C. Johnson, Matrix Analysis. Cambridge, U.K.: Cambridge Univ. Press, 2012.

[40] P. Shivakumar and K. Chew, "A sufficient condition for nonvanishing of determinants," Proc. Amer. Math. Soc., vol. 43, no. 1, pp. 63-66, Mar. 1974.

[41] Z. Li, Z. Duan, G. Chen, and L. Huang, "Consensus of multiagent systems and synchronization of complex networks: A unified viewpoint," IEEE Trans. Circuits Syst. I, Reg. Papers, vol. 57, no. 1, pp. 213-224, Jan. 2010.

[42] F. Lewis, H. Zhang, K. Hengster-Movric, and A. Das, Cooperative Control of Multi-Agent Systems: Optimal and Adaptive Design Approaches. London, U.K.: Springer-Verlag, 2014.

[43] R. S. Irving, Integers, Polynomials, and Rings: A Course in Algebra. Berlin, Germany: Springer-Verlag, 2004.

[44] J. Demmel, Applied Numerical Linear Algebra. Philadelphia, PA, USA: SIAM, 1997.

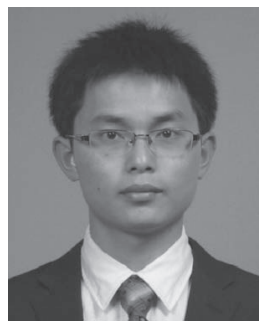

Yang Zheng received the B.E. degree from Tsinghua University, Beijing, China, in 2013. He is currently working toward the master's degree in automotive engineering with the College of Mechanical Engineering, Tsinghua University.

His research is on the topic of networked vehicular platoon control.

Mr. Zheng received the Best Student Paper Award at the 17th International IEEE Conference on Intelligent Transportation Systems, in 2014. He was the recipient of the National Scholarship and Outstanding Graduate in Tsinghua University.

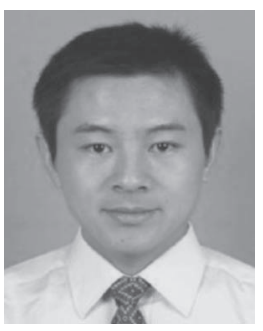

Shengbo Eben Li received the M.S. and Ph.D. degrees from Tsinghua University, Beijing, China, in 2006 and 2009, respectively.

From 2010 to 2012, he was a Postdoctoral Researcher with the University of Michigan, Ann Arbor, MI, USA. He is currently an Associate Professor with the Department of Automotive Engineering at Tsinghua University. His research interests include nonlinear dynamics and optimal control, autonomous vehicle control, driver assistance systems, and control of battery. He has authored or coauthored more than 60 peer-reviewed journal/conference papers, and he holds more than ten patents.

Dr. Li received of the Award for Science and Technology of China ITS Association (2012), the Award for Technological Invention in Ministry of Education (2012), the National Award for Technological Invention in China (2013), and the Honored Funding for Beijing Excellent Youth Researcher (2013).

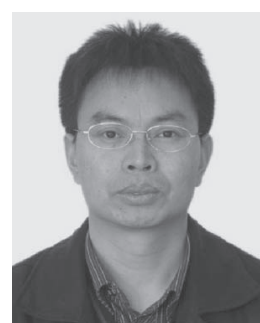

Jianqiang Wang received the B.Tech., M.S., and $\mathrm{Ph} . \mathrm{D}$. degrees from Jilin University of Technology, Jilin, China, in 1994, 1997, and 2002, respectively.

$\mathrm{He}$ is an Associate Professor with the Department of Automotive Engineering, Tsinghua University, Beijing, China. His research interests include intelligent vehicles, driving assistance systems, and driver behavior. He has authored over 40 journal papers and is a co-inventor on 30 patent applications.

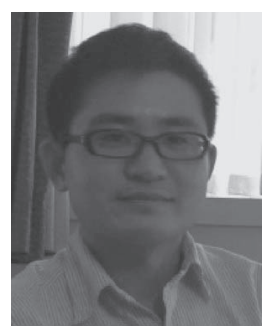

Dongpu Cao received the Ph.D. degree from Concordia University, Montreal, QC, Canada, in 2008. He is currently a Lecturer with the Center for Automotive Engineering, School of Engineering, Cranfield University, Bedford, U.K.

His research focuses mainly on electric and hybrid vehicles, vehicle dynamics and control, driver modeling, and intelligent vehicles, where he has contributed 4 co-edited Journal Special Editions, 1 patent, and about 70 publications.

Dr. Cao received the ASME AVTT'2010 Best Paper Award and the 2012 SAE Arch T. Colwell Merit Award. Dr. Cao serves as an Editor for IEEE TRANSACTIONS ON Vehicular TECHNOLOGY, an Associate Editor for IEEE TRANSACTIONS ON INDUSTRIAL ELECTRONICS, and an Editorial Board Member for another five journals. He has been a Guest Editor for Vehicle System Dynamics, IEEE/ASME TRANSACTIONS ON MECHATRONICS, and IEEE TRANSACTIONS ON INDUSTRIAL INFORMATICS. He serves on the SAE International Vehicle Dynamics Standards Committee and a few ASME, SAE, and IEEE technical committees.

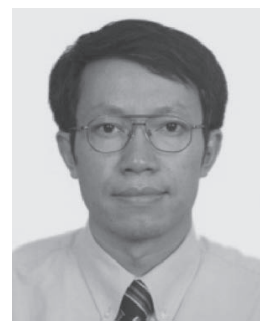

Keqiang $\mathbf{L i}$ received the B.Tech degree from Tsinghua University, Beijing, China, in 1985 and the M.S. and Ph.D. degrees from Chongqing University, Chongqing, China, in 1988 and 1995, respectively.

$\mathrm{He}$ is a Professor in automotive engineering at Tsinghua University. He has authored over 90 papers and is a co-inventor on 12 patents in China and Japan. His main areas of research interest include vehicle dynamics and control for driver assistance systems and hybrid electrical vehicle.

Dr. $\mathrm{Li}$ has served as a Senior Member of the Society of Automotive Engineers of China, and on the editorial boards of International Journal of ITS Research and International Journal of Vehicle Autonomous Systems. He has received the "Changjiang Scholar Program Professor" and some awards from public agencies and academic institutions of China. 
2015-03-06

\title{
Stability and scalability of homogeneous
} vehicular platoon: study on the influence of information flow topologies

\author{
Zheng, Yang \\ Institute of Electrical and Electronics Engineers
}

Yang Zheng, Shengbo Eben Li, Jianqiang Wang, Dongpu Cao and Keqiang Li. Stability and scalability of homogeneous vehicular platoon: study on the influence of information flow topologies. IEEE Transactions on Intelligent Transportation Systems, Volume: 17, Issue: 1, January 2016, pp14-26

http://dx.doi.org/10.1109/TITS.2015.2402153

Downloaded from Cranfield Library Services E-Repository 